KYUNGPOOK Math. J. 55(2015), 653-666

http://dx.doi.org/10.5666/KMJ.2015.55.3.653

pISSN 1225-6951 eISSN 0454-8124

(c) Kyungpook Mathematical Journal

\title{
Certain Nonlinear Differential Polynomials Sharing a Nonzero Polynomial with Finite Weight
}

\author{
ABhiJit BANERJEE* \\ Department of Mathematics, University of Kalyani, West Bengal-741235, India \\ e-mail : abanerjee_kal@yahoo.co.in and abanerjee_kal@rediffmail.com \\ Pulak SAHOO \\ Department of Mathematics, University of Kalyani, West Bengal-741235, India \\ e-mail : sahoopulak1@gmail.com and sahoopulak@yahoo.com
}

Abstract. With the notion of weighted sharing of values we study the uniqueness of meromorphic functions when certain nonlinear differential polynomials share a nonzero polynomial. Our results improve some recent results including that of a present first author [Kyungpook Math. J., 51(2011), 43-58].

\section{Introduction}

In this paper, by meromorphic functions we will always mean meromorphic functions in the complex plane. We adopt the standard notations in the Nevanlinna theory of meromorphic functions as explained in [6], [15] and [16]. It will be convenient to let $\mathrm{E}$ denote any set of positive real numbers of finite linear measure, not necessarily the same at each occurrence. For a nonconstant meromorphic function $h$, we denote by $T(r, h)$ the Nevanlinna characteristic of $h$ and by $S(r, h)$ any quantity satisfying $S(r, h)=o\{T(r, h)\}(r \rightarrow \infty, r \notin E)$.

Let $f$ and $g$ be two nonconstant meromorphic functions. For $a \in \mathbb{C} \cup\{\infty\}$ we say that $f$ and $g$ share the value $a$ CM (counting multiplicities) if $f-a$ and $g-a$ have the same set of zeros with the same multiplicities and we say that $f$ and $g$ share the value $a$ IM (ignoring multiplicities) if we do not consider the multiplicities.

\footnotetext{
* Corresponding Author.

Received June 11, 2013; accepted October 14, 2013.

2010 Mathematics Subject Classification: Primary 30D35.

Key words and phrases: Meromorphic function, Weighted Sharing, Nonlinear differential polynomials.

Both the authors are thankful to DST-PURSE programme for financial assistance.
} 
Throughout this paper, we need the following definition.

$$
\Theta(a, f)=1-\limsup _{r \longrightarrow \infty} \frac{\bar{N}(r, a ; f)}{T(r, f)},
$$

where $a$ is a value in the extended complex plane.

In 1959, Hayman [5] proved the following result.

Theorem A. Let $f$ be a transcendental entire function, and let $n(\geq 1)$ be an integer. Then $f^{n} f^{\prime}=1$ has infinitely many zeros.

In 2002, Fang and Fang [4] proved the following result.

Theorem B. Let $f$ and $g$ be two nonconstant entire functions, and let $n(\geq 8)$ be an integer. If $f^{n}(f-1) f^{\prime}$ and $g^{n}(g-1) g^{\prime}$ share $1 C M$, then $f \equiv g$.

In the same year Fang [3] investigated the value sharing of more general nonlinear differential polynomial than that was considered in Theorem B and obtained the following result.

Theorem C. Let $f$ and $g$ be two nonconstant entire functions, and let $n, k$ be two positive integers with $n \geq 2 k+8$. If $\left[f^{n}(f-1)\right]^{(k)}$ and $\left[g^{n}(g-1)\right]^{(k)}$ share $1 C M$, then $f \equiv g$.

In 2004, Lin and Yi [12] considered the case of meromorphic function in Theorem $\mathrm{B}$ and obtained the following.

Theorem D. Let $f$ and $g$ be two nonconstant meromorphic functions with $\Theta(\infty, f)>2 /(n+1)$, and let $n(\geq 12)$ be an integer. If $f^{n}(f-1) f^{\prime}$ and $g^{n}(g-1) g^{\prime}$ share $1 C M$, then $f \equiv g$.

Natural inquisition would be to investigate the situation for meromorphic function in Theorem C. In this direction in 2008, Zhang [17] proved the following result.

Theorem E. Suppose that $f$ is a transcendental meromorphic function with finite number of poles, $g$ is a transcendental entire function, and let $n, k$ be two positive integers with $n \geq 2 k+6$. If $\left(f^{n}(f-1)\right)^{(k)}$ and $\left(g^{n}(g-1)\right)^{(k)}$ share $1 C M$, then $f \equiv g$.

To proceed further we require the following definition known as weighted sharing of values introduced by I. Lahiri [7] which measure how close a shared value is to being shared CM or to being shared IM.

Definition 1.1. Let $k$ be a nonnegative integer or infinity. For $a \in \mathbb{C} \cup\{\infty\}$ we denote by $E_{k}(a ; f)$ the set of all $a$-points of $f$ where an $a$-point of multiplicity $\mathrm{m}$ is counted $\mathrm{m}$ times if $m \leq k$ and $\mathrm{k}+1$ times if $m>k$. If $E_{k}(a ; f)=E_{k}(a ; g)$, we say that $f, g$ share the value $a$ with weight $\mathrm{k}$.

The definition implies that if $f, g$ share a value $a$ with weight $k$, then $z_{0}$ is an $a$-point of $f$ with multiplicity $m(\leq k)$ if and only if it is an $a$-point of $g$ with multiplicity $m(\leq k)$ and $z_{0}$ is an a-point of $f$ with multiplicity $m(>k)$ if and only 
if it is an a-point of $g$ with multiplicity $n(>k)$, where $\mathrm{m}$ is not necessarily equal to n.

We write $f, g$ share $(a, k)$ to mean that $f, g$ share the value $a$ with weight $\mathrm{k}$. Clearly if $f, g$ share $(a, k)$ then $f, g$ share $(a, p)$ for any integer $p, 0 \leq p<k$. Also we note that $f, g$ share a value $a$ IM or CM if and only if $f, g$ share $(a, 0)$ or $(a, \infty)$ respectively.

In 2009, using the notion of weighted sharing of values, $\mathrm{Xu}$, Yi and Cao [13] proved the following result.

Theorem $\mathbf{F}$. Let $f$ and $g$ be two nonconstant meromorphic functions, and $n(\geq 1)$, $k(\geq 1)$ and $l(\geq 0)$ be three integers such that $\Theta(\infty, f)+\Theta(\infty, g)>4 / n$. Suppose $\left[f^{n}(f-1)\right]^{(k)}$ and $\left[g^{n}(g-1)\right]^{(k)}$ share $(1, l)$. If $l \geq 2$ and $n>5 k+11$ or if $l=1$ and $n>7 k+23 / 2$, then $f=g$.

Note 1.1. The above result is partially correct. According to the method of approach in [13] there is a gap in the proof of Theorem 1.2.

Recently, Li [11] proved the following result which rectify and at the same time improve Theorem F.

Theorem G. Let $f$ and $g$ be two nonconstant meromorphic functions, and $n(\geq 1)$, $k(\geq 1)$ and $l(\geq 0)$ be three integers such that $\Theta(\infty, f)+\Theta(\infty, g)>4 / n$. Suppose $\left[f^{n}(f-1)\right]^{(k)}$ and $\left[g^{n}(g-1)\right]^{(k)}$ share $(1, l)$. If $l \geq 2$ and $n>3 k+11$ or if $l=1$ and $n>5 k+14$, then $f=g$ or $\left[f^{n}(f-1)\right]^{(k)}\left[g^{n}(g-1)\right]^{(k)}=1$.

Note 1.2. Unfortunately Theorem $G$ is also not true for rational functions. One can easily see that in the proof of Lemma 2.5 in subcase 3.2, $(2.22)$ is only true when $f$ and $g$ are transcendental functions. Since in the proof of Theorem G, Lemma 2.5 plays a vital role, the same theorem cease to be hold for rational functions.

In this direction recently the present first author [1] proved the following results first one of which improves Theorem G.

Theorem H. Let $f$ and $g$ be two transcendental meromorphic functions and $n(\geq 1)$, $k(\geq 1), l(\geq 0)$ be three integers such that $\Theta(\infty, f)+\Theta(\infty, g)>4 / n$. Suppose for two nonzero constants $a$ and $b,\left[f^{n}(a f+b)\right]^{(k)}$ and $\left[g^{n}(a g+b)\right]^{(k)}$ share $(1, l)$. If $l \geq 2$ and $n \geq 3 k+9$ or if $l=1$ and $n \geq 4 k+10$ or if $l=0$ and $n \geq 9 k+18$, then $f=g$ or $\left[f^{n}(a f+b)\right]^{(k)}\left[g^{n}(a g+b)\right]^{(k)}=1$. The possibility $\left[f^{n}(a f+b)\right]^{(k)}\left[g^{n}(a g+b)\right]^{(k)}=1$ does not occur for $k=1$.

Theorem I. Let $f$ and $g$ be two transcendental entire functions, and let $n(\geq 1), k(\geq$ $1), l(\geq 0)$ be three integers. Suppose for two nonzero constants $a$ and $b,\left[f^{n}(a f+\right.$ $b)]^{(k)}$ and $\left[g^{n}(a g+b)\right]^{(k)}$ share $(1, l)$. If $l \geq 2$ and $n \geq 2 k+6$ or if $l=1$ and $n \geq 5 k / 2+7$ or if $l=0$ and $n \geq 5 k+12$, then $f=g$.

Following questions are inevitable:

Question 1.1. Is it possible in any way to remove the second conclusion of Theorem $\mathrm{H}$ ? 
Question 1.2. What can be said if one replace the sharing value 1 by a nonzero polynomial in Theorems $\mathrm{H}$ and I?

In the paper, our main concern is to find the affirmative answer of the above questions. We prove following two theorems first one of which improves Theorem $\mathrm{H}$ and second one improves Theorem I. We now state the main results of the paper.

Theorem 1.1. Let $f$ and $g$ be two non-entire transcendental meromorphic functions, and let $n(\geq 1), k(\geq 1), l(\geq 0)$ be three integers such that $\Theta(\infty, f)+$ $\Theta(\infty, g)>4 / n$. Suppose for two nonzero constants a and $b,\left[f^{n}(a f+b)\right]^{(k)}-P$ and $\left[g^{n}(a g+b)\right]^{(k)}-P$ share $(0, l)$ where $P(\not \equiv 0)$ is a polynomial. If $l \geq 2$ and $n \geq 3 k+9$ or if $l=1$ and $n \geq 4 k+10$ or if $l=0$ and $n \geq 9 k+18$, then $f=g$.

Theorem 1.2. Let $f$ and $g$ be two transcendental entire functions, and let $n(\geq$ $1), k(\geq 1), l(\geq 0)$ be three integers. Suppose for two nonzero constants $a$ and $b$, $\left[f^{n}(a f+b)\right]^{(k)}-P$ and $\left[g^{n}(a g+b)\right]^{(k)}-P$ share $(0, l)$ where $P(\not \equiv)$ is a polynomial. If $l \geq 2$ and $n \geq 2 k+6$ or if $l=1$ and $n \geq 5 k / 2+7$ or if $l=0$ and $n \geq 5 k+12$, then $f=g$.

However the following question is still open :

Question 1.3. Keeping all other conditions intact, are Theorems 1.1 and 1.2 true for rational functions also?

Though the standard definitions and notations of the value distribution theory are available in [6], we explain some definitions and notations which are used in the paper.

Definition 1.2.([8]) For $a \in \mathbb{C} \cup\{\infty\}$ we denote by $N(r, a ; f \mid=1)$ the counting function of simple $a$ points of $f$. For a positive integer $p$ we denote by $N(r, a ; f \mid \leq p)$ the counting function of those $a$-points of $f$ (counted with multiplicities) whose multiplicities are not greater than $p$. By $\bar{N}(r, a ; f \mid \leq p)$ we denote the corresponding reduced counting function.

In an analogous manner we define $N(r, a ; f \mid \geq p)$ and $\bar{N}(r, a ; f \mid \geq p)$.

Definition 1.3.([7]) Let $k$ be a positive integer or infinity. We denote by $N_{k}(r, a ; f)$ the counting function of $a$-points of $f$, where an $a$-point of multiplicity $m$ is counted $m$ times if $m \leq k$ and $k$ times if $m>k$. Then

$$
N_{k}(r, a ; f)=\bar{N}(r, a ; f)+\bar{N}(r, a ; f \mid \geq 2)+\ldots+\bar{N}(r, a ; f \mid \geq k) .
$$

\section{Lemmas}

In this section we present some lemmas which will be needed in the sequel. Let $F$ and $G$ be two nonconstant meromorphic functions defined in $\mathbb{C}$. We shall denote by $H$ the following function:

$$
H=\left(\frac{F^{\prime \prime}}{F^{\prime}}-\frac{2 F^{\prime}}{F-1}\right)-\left(\frac{G^{\prime \prime}}{G^{\prime}}-\frac{2 G^{\prime}}{G-1}\right) .
$$


Lemma 2.1.([14]) Let $f$ be a transcendental meromorphic function, and let $P_{n}(f)$ be a differential polynomial in $f$ of the form

$$
P_{n}(f)=a_{n} f^{n}(z)+a_{n-1} f^{n-1}(z)+\ldots+a_{1} f(z)+a_{0},
$$

where $a_{n}(\neq 0), a_{n-1}, \ldots, a_{1}, a_{0}$ are complex numbers. Then

$$
T\left(r, P_{n}(f)\right)=n T(r, f)+O(1) .
$$

Lemma 2.2.([18]) Let $f$ be a nonconstant meromorphic function, and $p, k$ be positive integers. Then

$$
\begin{gathered}
N_{p}\left(r, 0 ; f^{(k)}\right) \leq T\left(r, f^{(k)}\right)-T(r, f)+N_{p+k}(r, 0 ; f)+S(r, f), \\
N_{p}\left(r, 0 ; f^{(k)}\right) \leq k \bar{N}(r, \infty ; f)+N_{p+k}(r, 0 ; f)+S(r, f) .
\end{gathered}
$$

Lemma 2.3.([7]) Let $F$ and $G$ be two nonconstant meromorphic functions sharing $(1,2)$. Then one of the following cases holds:

(i) $T(r) \leq N_{2}(r, 0 ; F)+N_{2}(r, 0 ; G)+N_{2}(r, \infty ; F)+N_{2}(r, \infty ; G)+S(r)$,

(ii) $F=G$,

(iii) $F G=1$,

where $T(r)$ denotes the maximum of $T(r, F)$ and $T(r, G)$ and $S(r)=o\{T(r)\}$ as $r \rightarrow \infty$, possibly outside a set of finite linear measure.

Lemma 2.4.([2]) Let $F$ and $G$ be two nonconstant meromorphic functions sharing $(1,1)$ and $H \not \equiv 0$. Then

$T(r, F) \leq N_{2}(r, 0 ; F)+N_{2}(r, 0 ; G)+N_{2}(r, \infty ; F)+N_{2}(r, \infty ; G)+\frac{1}{2} \bar{N}(r, 0 ; F)+$ $\frac{1}{2} \bar{N}(r, \infty ; F)+S(r, F)+S(r, G)$.

Lemma 2.5.([2]) Let $F$ and $G$ be two nonconstant meromorphic functions sharing $(1,0)$ and $H \not \equiv 0$. Then

$T(r, F) \leq N_{2}(r, 0 ; F)+N_{2}(r, 0 ; G)+N_{2}(r, \infty ; F)+N_{2}(r, \infty ; G)+2 \bar{N}(r, 0 ; F)+$ $\bar{N}(r, 0 ; G)+2 \bar{N}(r, \infty ; F)+\bar{N}(r, \infty ; G)+S(r, F)+S(r, G)$.

Lemma 2.6.([10]) If $N\left(r, 0 ; f^{(k)} \mid f \neq 0\right)$ denote the counting function of those zeros of $f^{(k)}$ which are not the zeros of $f$, where a zero of $f^{(k)}$ is counted according to its multiplicity, then

$N\left(r, 0 ; f^{(k)} \mid f \neq 0\right) \leq k \bar{N}(r, \infty ; f)+N(r, 0 ; f \mid<k)+k \bar{N}(r, 0 ; f \mid \geq k)+S(r, f)$.

Lemma 2.7.([6],[15]) Let $f$ be a transcendental meromorphic function, and let $a_{1}(z), a_{2}(z)$ be two distinct meromorphic functions such that $T\left(r, a_{i}(z)\right)=S(r, f)$, $i=1$,2. Then

$$
T(r, f) \leq \bar{N}(r, \infty ; f)+\bar{N}\left(r, a_{1} ; f\right)+\bar{N}\left(r, a_{2} ; f\right)+S(r, f) .
$$


Lemma 2.8. Let $f$ and $g$ be two non-entire transcendental meromorphic functions, let $n, k$ be two positive integers and let $P$ be a nonconstant polynomial. If $n \geq 2 k+5$, then

$$
\left(f^{n}(a f+b)\right)^{(k)}\left(g^{n}(a g+b)\right)^{(k)} \neq P^{2},
$$

where $a, b$ are any two nonzero constants.

Proof. If possible, let

$$
\left(f^{n}(a f+b)\right)^{(k)}\left(g^{n}(a g+b)\right)^{(k)}=P^{2} .
$$

Let $z_{1} \notin\{z: P(z)=0\}$ be a zero of $f$ with multiplicity $p_{1}(\geq 1)$. Then it follows from (2.3) that $z_{1}$ is a pole of $g$. Suppose that $z_{1}$ is a pole of $g$ of order $q_{1}(\geq 1)$. Then we have

$$
n p_{1}-k=(n+1) q_{1}+k .
$$

From (2.4) we get $q_{1}+2 k=n\left(p_{1}-q_{1}\right) \geq n$, i.e., $q_{1} \geq n-2 k$. Thus from (2.4) we obtain $n p_{1}=(n+1) q_{1}+2 k$, and so

$$
p_{1} \geq n-2 k+1 \text {. }
$$

Let $z_{2} \notin\{z: P(z)=0\}$ be a zero of $a f+b$ with multiplicity $p_{2}(\geq k+1)$. Then from (2.3) it follows that $z_{2}$ is a pole of $g$. Suppose that $z_{2}$ is a pole of $g$ of order $q_{2}(\geq 1)$. Then we have $p_{2}-k=(n+1) q_{2}+k$, i.e.,

$$
p_{2} \geq n+2 k+1
$$

If $z_{3} \notin\{z: P(z)=0\}$ is a zero of $a f+b$ with multiplicity $p_{3}(\leq k)$, then from (2.3) it follows that $z_{3}$ may be a zero of $\left(f^{n}(a f+b)\right)^{k}$ and if it happens then it will be a pole of $g$ with multiplicity $n+k+1$. Suppose that $z_{4} \notin\{z: P(z)=0\}$ be a pole of $f$. Then from (2.3) it is clear that $z_{4}$ is either a zero of $g^{n}(a g+b)$ or a zero of $\left(g^{n}(a g+b)\right)^{(k)}$. Therefore

$$
\begin{aligned}
\bar{N}(r, \infty ; f) \leq & \bar{N}(r, 0 ; g)+\bar{N}(r, 0 ; a g+b \mid \leq k)+\bar{N}(r, 0 ; a g+b \mid \geq k+1) \\
& +\bar{N}\left(r, 0 ; h^{(k)} \mid h \neq 0\right)+S(r, g),
\end{aligned}
$$

where $\bar{N}\left(r, 0 ; h^{(k)} \mid h \neq 0\right)$ denotes the reduced counting function of those zeros of $h^{(k)}$ that are not the zeros of $h$ and $h=g^{n}(a g+b)$. 
By Lemma 2.6 we have

$$
\begin{aligned}
\bar{N}\left(r, 0 ; h^{(k)} \mid h \neq 0\right) \leq & \frac{1}{n+k+1}\left[N\left(r, 0 ; h^{(k)} \mid h \neq 0\right)\right] \\
\leq & \frac{1}{n+k+1}[k \bar{N}(r, \infty ; h)+N(r, 0 ; h \mid<k)+k \bar{N}(r, 0 ; h \mid \geq k)] \\
\leq & \frac{1}{n+k+1}\left[k \bar{N}(r, \infty ; h)+N_{k}(r, 0 ; h)\right] \\
\leq & \frac{k}{n+k+1}[\bar{N}(r, \infty ; g)+\bar{N}(r, 0 ; g)+\bar{N}(r, 0 ; a g+b)] \\
\leq & \frac{k}{n+k+1}[\bar{N}(r, \infty ; g)+\bar{N}(r, 0 ; g)+\bar{N}(r, 0 ; a g+b \mid \leq k) \\
& +\bar{N}(r, 0 ; a g+b \mid \geq k+1)] .
\end{aligned}
$$

So from (2.5) we obtain

$$
\begin{aligned}
\bar{N}(r, \infty ; f) \leq & \left(1+\frac{k}{n+k+1}\right) \\
& {[\bar{N}(r, 0 ; g)+\bar{N}(r, 0 ; a g+b \mid \leq k)+\bar{N}(r, 0 ; a g+b \mid \geq k+1)] } \\
& +\frac{k}{n+k+1} \bar{N}(r, \infty ; g)+S(r, g) \\
\leq & \frac{n+2 k+1}{n+k+1}\left[\frac{1}{n-2 k+1}+\frac{1}{n+k+1}+\frac{1}{n+2 k+1}\right] T(r, g) \\
& +\frac{k}{n+k+1} T(r, g)+S(r, g) \\
\leq & \left(\frac{2(n+1)}{(n+k+1)(n-2 k+1)}+\frac{k}{(n+k+1)^{2}}+\frac{k+1}{n+k+1}\right) \\
& T(r, g)+S(r, g) .
\end{aligned}
$$

Using the second fundamental theorem of Nevanlinna we get

$$
\begin{aligned}
T(r, f) \leq & \bar{N}(r, \infty ; f)+\bar{N}(r, 0 ; f)+\bar{N}(r, 0 ; a f+b)+S(r, f) \\
\leq & \bar{N}(r, \infty ; f)+\bar{N}(r, 0 ; f)+\bar{N}(r, 0 ; a f+b \mid \leq k) \\
& +\bar{N}(r, 0 ; a f+b \mid \geq k+1)+S(r, f) \\
\leq & {\left[\frac{2(n+1)}{(n+k+1)(n-2 k+1)}+\frac{k}{(n+k+1)^{2}}+\frac{k+1}{n+k+1}\right] T(r, g) } \\
& +\left[\frac{2(n+1)}{(n+2 k+1)(n-2 k+1)}+\frac{1}{n+k+1}\right] T(r, f)+S(r, f)+S(r, g) .
\end{aligned}
$$

Similarly

$$
\begin{aligned}
T(r, g) \leq & {\left[\frac{2(n+1)}{(n+k+1)(n-2 k+1)}+\frac{k}{(n+k+1)^{2}}+\frac{k+1}{n+k+1}\right] T(r, f) } \\
(2.7) \leq & +\left[\frac{2(n+1)}{(n+2 k+1)(n-2 k+1)}+\frac{1}{n+k+1}\right] T(r, g)+S(r, f)+S(r, g) .
\end{aligned}
$$


Adding (2.6) and (2.7) we obtain

$$
\begin{array}{r}
{\left[\frac{n-1}{n+k+1}-\frac{2(n+1)(2 n+3 k+2)}{(n-2 k+1)(n+2 k+1)(n+k+1)}-\frac{k}{(n+k+1)^{2}}\right]} \\
\{T(r, f)+T(r, g)\} \leq S(r, f)+S(r, g),
\end{array}
$$

i.e.,

$$
\begin{array}{r}
{[(n-1)(n+k+1)(n-2 k+1)(n+2 k+1)-2(n+1)(n+k+1)(2 n+3 k+2)} \\
-k(n-2 k+1)(n+2 k+1)]\{T(r, f)+T(r, g)\} \leq S(r, f)+S(r, g),
\end{array}
$$

contradicting with the fact that $n \geq 2 k+5$. This proves the lemma.

Lemma 2.9. Let $f$ and $g$ be two transcendental entire function, let $n, k$ are any two positive integers and let $P$ be a nonconstant polynomial. Then

$$
\left(f^{n}(a f+b)\right)^{(k)}\left(g^{n}(a g+b)\right)^{(k)} \neq P^{2},
$$

where $a, b$ are any two nonzero constants.

Proof. Suppose that

$$
\left(f^{n}(a f+b)\right)^{(k)}\left(g^{n}(a g+b)\right)^{(k)}=P^{2} .
$$

Let $z_{0}$ be a zero of $f$ with multiplicity $p$. Then clearly $z_{0}$ is a zero of $P$. Since $P$ is a polynomial, $f$ has a finite number of zeros. So we put $f(z)=P_{1} e^{\alpha}$, where $\alpha$ is a nonconstant entire function and $P_{1}$ is a polynomial. Now

$$
\begin{gathered}
\left(a f^{n+1}\right)^{(k)}=t_{1}\left(\alpha^{\prime}, \alpha^{\prime \prime}, \ldots, \alpha^{(k)}, P_{1}\right) e^{(n+1) \alpha}, \\
\left(b f^{n}\right)^{(k)}=t_{0}\left(\alpha^{\prime}, \alpha^{\prime \prime}, \ldots, \alpha^{(k)}, P_{1}\right) e^{n \alpha},
\end{gathered}
$$

where $t_{i}\left(\alpha^{\prime}, \alpha^{\prime \prime}, \ldots, \alpha^{(k)}, P_{1}\right)(i=0,1)$ are differential polynomials in $\alpha^{\prime}, \alpha^{\prime \prime}, \ldots$, $\alpha^{(k)}$ with coefficients which are rational functions in $P_{1}$ or its derivatives. Obviously

$$
t_{i}\left(\alpha^{\prime}, \alpha^{\prime \prime}, \ldots, \alpha^{(k)}, P_{1}\right) \neq 0
$$

for $i=0,1$, and

$$
\left(f^{n}(a f+b)\right)^{(k)} \neq 0 .
$$

From (2.8) and (2.9) we have

$$
t_{1}\left(\alpha^{\prime}, \alpha^{\prime \prime}, \ldots, \alpha^{(k)}, P_{1}\right) e^{\alpha(z)}+t_{0}\left(\alpha^{\prime}, \alpha^{\prime \prime}, \ldots, \alpha^{(k)}, P_{1}\right) \neq 0 .
$$

Since $\alpha(z)$ is an entire function, we obtain $T\left(r, \alpha^{(j)}\right)=S(r, f)$ for $j=1,2, \ldots, k$. Thus $T\left(r, t_{i}\right)=S(r, f)$ for $i=0,1$. So from (2.10), Lemma 2.1 and Lemma 2.7 we obtain

$$
\begin{aligned}
T(r, f) & =T\left(r, t_{1} e^{\alpha}\right)+S(r, f) \\
& \leq \bar{N}\left(r, 0 ; t_{1} e^{\alpha}\right)+\bar{N}\left(r, 0 ; t_{1} e^{\alpha}+t_{0}\right)+S(r, f) \\
& \leq S(r, f),
\end{aligned}
$$


which is a contradiction. This completes the proof of the lemma.

Lemma 2.10. Let $f$ and $g$ be two transcendental meromorphic (entire) functions and let $n(\geq 1), k(\geq 1)$, be two integers. Suppose that $F=\frac{\left(f^{n}(a f+b)\right)^{(k)}}{P(z)}$ and $G=$ $\frac{\left(g^{n}(a g+b)\right)^{(k)}}{P(z)}$. If there exist two nonzero constants $c_{1}$ and $c_{2}$ such that $\bar{N}\left(r, c_{1} ; F\right)=$ $\bar{N}(r, 0 ; G)$ and $\bar{N}\left(r, c_{2} ; G\right)=\bar{N}(r, 0 ; F)$, then $n \leq 3 k+4(n \leq 2 k+3)$.

Proof. We prove the theorem for two transcendental meromorphic functions. By the second fundamental theorem of Nevanlinna we have

$$
\begin{aligned}
T(r, F) & \leq \bar{N}(r, 0 ; F)+\bar{N}(r, \infty ; F)+\bar{N}\left(r, c_{1} ; F\right)+S(r, F) \\
& \leq \bar{N}(r, 0 ; F)+\bar{N}(r, 0 ; G)+\bar{N}(r, \infty ; F)+S(r, F) .
\end{aligned}
$$

By (2.1), (2.2), (2.11) and Lemma 2.1 we obtain

$$
\begin{aligned}
(n+1) T(r, f) \leq & T(r, F)-\bar{N}(r, 0 ; F)+N_{k+1}\left(r, 0 ; f^{n}(a f+b)\right) \\
& +O\{\log r\}+S(r, f) \\
\leq & \bar{N}(r, 0 ; G)+N_{k+1}\left(r, 0 ; f^{n}(a f+b)\right)+\bar{N}(r, \infty ; f) \\
& +O\{\log r\}+S(r, f) \\
\leq & N_{k+1}\left(r, 0 ; f^{n}(a f+b)\right)+N_{k+1}\left(r, 0 ; g^{n}(a g+b)\right)+\bar{N}(r, \infty ; f) \\
& +k \bar{N}(r, \infty ; g)+O\{\log r\}+S(r, f)+S(r, g) \\
\leq & (k+3) T(r, f)+(2 k+2) T(r, g)+O\{\log r\} \\
& +S(r, f)+S(r, g) .
\end{aligned}
$$

Similarly we obtain

$$
\begin{aligned}
(n+1) T(r, g) \leq & (k+3) T(r, g)+(2 k+2) T(r, f)+O\{\log r\} \\
& +S(r, f)+S(r, g) .
\end{aligned}
$$

Combining (2.12), (2.13) and noting that $O\{\log r\}=o(T(r, f))$ and $O\{\log r\}=$ $o(T(r, g))$ we get

$$
(n-3 k-4)\{T(r, f)+T(r, g)\} \leq S(r, f)+S(r, g),
$$

which gives $n \leq 3 k+4$. This completes the proof of the lemma.

Lemma 2.11. Let $f$ and $g$ be two nonconstant meromorphic functions such that

$$
\Theta(\infty, f)+\Theta(\infty, g)>\frac{4}{n},
$$

where $n(\geq 3)$ is an integer. Then

$$
f^{n}(a f+b)=g^{n}(a g+b)
$$

implies $f=g$, where $a, b$ are two nonzero constants. 
Proof. We omit the proof since it can be carried out in the line of Lemma 6 [9].

\section{Proof of Theorems}

Proof of Theorem 1.1. Let $F(z)$ and $G(z)$ be given as in Lemma 2.10. Then $F(z), G(z)$ are non-entire transcendental meromorphic functions that share $(1, l)$ except the the zeros of the polynomial $P(z)$. So from (2.1) we obtain

$$
\begin{aligned}
N_{2}(r, 0 ; F) \leq & N_{2}\left(r, 0 ;\left(f^{n}(a f+b)\right)^{(k)}\right)+S(r, f) \\
\leq & T\left(r,\left(f^{n}(a f+b)\right)^{(k)}\right)-(n+1) T(r, f) \\
& +N_{k+2}\left(r, 0 ; f^{n}(a f+b)\right)+S(r, f) \\
\leq \quad & T(r, F)-(n+1) T(r, f)+N_{k+2}\left(r, 0 ; f^{n}(a f+b)\right) \\
& +O\{\log r\}+S(r, f) .
\end{aligned}
$$

Again by $(2.2)$ we have

$$
N_{2}(r, 0 ; G) \leq k \bar{N}(r, \infty ; f)+N_{k+2}\left(r, 0 ; g^{n}(a g+b)\right)+S(r, g) .
$$

From (3.1) we get

$$
\begin{aligned}
(n+1) T(r, f) \leq & T(r, F)+N_{k+2}\left(r, 0 ; f^{n}(a f+b)\right)-N_{2}(r, 0 ; F) \\
& +O\{\log r\}+S(r, f) .
\end{aligned}
$$

Now we consider the following three cases.

Case 1. Let $l \geq 2$. Let (i) of Lemma 2.3 holds. Then using (3.2) we obtain from (3.3)

$$
\begin{aligned}
(n+1) T(r, f) \leq & N_{2}(r, 0 ; G)+N_{2}(r, \infty ; F)+N_{2}(r, \infty ; G)+N_{k+2}\left(r, 0 ; f^{n}(a f+b)\right) \\
& +O\{\log r\}+S(r, f)+S(r, g) \\
\leq & N_{k+2}\left(r, 0 ; f^{n}(a f+b)\right)+N_{k+2}\left(r, 0 ; g^{n}(a g+b)\right)+2 \bar{N}(r, \infty ; f) \\
& +(k+2) \bar{N}(r, \infty ; g)+O\{\log r\}+S(r, f)+S(r, g) \\
\leq & (k+3)\{T(r, f)+T(r, g)\}+2 \bar{N}(r, \infty ; f)+(k+2) \bar{N}(r, \infty ; g) \\
& +O\{\log r\}+S(r, f)+S(r, g) \\
\leq & {[k+5-2 \Theta(\infty, f)+\varepsilon] T(r, f)+[2 k+5-(k+2) \Theta(\infty, g)+\varepsilon] } \\
& T(r, g)+S(r, f)+S(r, g) \\
\leq & {[3 k+10-2 \Theta(\infty, f)-2 \Theta(\infty, g)-k \min \{\Theta(\infty, f), \Theta(\infty, g)\}} \\
& +2 \varepsilon] T(r)+S(r) .
\end{aligned}
$$

In a similar way we can obtain

$$
\begin{aligned}
(n+1) T(r, g) \leq & {[3 k+10-2 \Theta(\infty, f)-2 \Theta(\infty, g)-k \min \{\Theta(\infty, f), \Theta(\infty, g)\}} \\
& +2 \varepsilon] T(r)+S(r) .
\end{aligned}
$$


From (3.4) and (3.5) we obtain

$[n-3 k-9+2 \Theta(\infty, f)+2 \Theta(\infty, g)+k \min \{\Theta(\infty, f), \Theta(\infty, g)\}-2 \varepsilon] T(r) \leq S(r)$,

contradicting with the facts that $n \geq 3 k+9, \Theta(\infty, f)+\Theta(\infty, g)>4 / n$ and $\varepsilon>0$ be arbitrary. So by Lemma 2.3 either $F G=1$ or $F=G$. Let $F G=1$. Then

$$
\left(f^{n}(a f+b)\right)^{(k)}\left(g^{n}(a g+b)\right)^{(k)}=P^{2},
$$

a contradiction by Lemma 2.8. So we have $F=G$. That is

$$
\left[f^{n}(a f+b)\right]^{(k)}=\left[g^{n}(a g+b)\right]^{(k)} .
$$

Integrating we get

$$
\left[f^{n}(a f+b)\right]^{(k-1)}=\left[g^{n}(a g+b)\right]^{(k-1)}+c_{k-1},
$$

where $c_{k-1}$ is a constant. If $c_{k-1} \neq 0$, from Lemma 2.10 we obtain $n \leq 3 k+4$, a contradiction. Hence $c_{k-1}=0$. Repeating k-times, we obtain

$$
f^{n}(a f+b)=g^{n}(a g+b) .
$$

Now the result follows from Lemma 2.11.

Case 2. Let $l=1$ and $H \not \equiv 0$. Using Lemma 2.4 and (3.2) we obtain from (3.3)

$$
\begin{aligned}
(n+1) T(r, f) \leq & N_{2}(r, 0 ; G)+N_{2}(r, \infty ; F)+N_{2}(r, \infty ; G)+\frac{1}{2} \bar{N}(r, 0 ; F) \\
& +\frac{1}{2} \bar{N}(r, \infty ; F)+N_{k+2}\left(r, 0 ; f^{n}(a f+b)\right) \\
& +O\{\log r\}+S(r, f)+S(r, g) \\
\leq \quad & N_{k+2}\left(r, 0 ; f^{n}(a f+b)\right)+N_{k+2}\left(r, 0 ; g^{n}(a g+b)\right) \\
& +\frac{1}{2} N_{k+1}\left(r, 0 ; f^{n}(a f+b)\right)+\frac{k+5}{2} \bar{N}(r, \infty ; f) \\
& +(k+2) \bar{N}(r, \infty ; g))+O\{\log r\}+S(r, f)+S(r, g) \\
\leq & {\left[2 k+\frac{13}{2}-\left(\frac{k}{2}+2\right) \Theta(\infty, f)-\frac{1}{2} \Theta(\infty, f)+\varepsilon\right] T(r, f) } \\
& \left.+\left[2 k+5-\left(\frac{k}{2}+2\right) \Theta(\infty, g)-\frac{k}{2} \Theta(\infty, g)+\varepsilon\right] T(r, g)\right\} \\
& +O\{\log r\}+S(r, f)+S(r, g) \\
\leq & {\left[4 k+\frac{23}{2}-\frac{k+5}{2}(\Theta(\infty, f)+\Theta(\infty, g))+2 \varepsilon\right] T(r)+S(r) . }
\end{aligned}
$$

Similarly

$$
\begin{aligned}
(n+1) T(r, g) \leq & {\left[4 k+\frac{23}{2}-\frac{k+5}{2}(\Theta(\infty, f)+\Theta(\infty, g))+2 \varepsilon\right] T(r) } \\
& +S(r) .
\end{aligned}
$$


Combining (3.7) and (3.8) we obtain

$$
\left[n-4 k-\frac{21}{2}+\frac{k+5}{2}(\Theta(\infty, f)+\Theta(\infty, g))-2 \varepsilon\right] T(r) \leq S(r),
$$

a contradiction since $n \geq 4 k+10, \Theta(\infty, f)+\Theta(\infty, g)>4 / n$ and $\varepsilon>0$ be arbitrary. We now assume that $H=0$. That is

$$
\left(\frac{F^{\prime \prime}}{F^{\prime}}-\frac{2 F^{\prime}}{F-1}\right)-\left(\frac{G^{\prime \prime}}{G^{\prime}}-\frac{2 G^{\prime}}{G-1}\right)=0 .
$$

Integrating both sides of the above equality twice we get

$$
\frac{1}{F-1}=\frac{A}{G-1}+B
$$

where $A(\neq 0)$ and $B$ are constants. From (3.9) it is clear that $F, G$ share the value $1 \mathrm{CM}$ and so they share 1 with weight two. Hence we have $n \geq 3 k+9$. Now we discuss the following three subcases.

Subcase 1. Let $B \neq 0$ and $A=B$. Then from (3.9) we get

$$
\frac{1}{F-1}=\frac{B G}{G-1} \text {. }
$$

If $B=-1$, then from (3.10) we obtain

$$
F G=1,
$$

a contradiction by Lemma 2.8 .

If $B \neq-1$, from (3.10), we have $\frac{1}{F}=\frac{B G}{(1+B) G-1}$ and so $\bar{N}\left(r, \frac{1}{1+B} ; G\right)=\bar{N}(r, 0 ; F)$.

Now from the second fundamental theorem of Nevanlinna, we get

$$
\begin{aligned}
T(r, G) & \leq \bar{N}(r, 0 ; G)+\bar{N}\left(r, \frac{1}{1+B} ; G\right)+\bar{N}(r, \infty ; G)+S(r, G) \\
& \leq \bar{N}(r, 0 ; F)+\bar{N}(r, 0 ; G)+\bar{N}(r, \infty ; G)+S(r, G) .
\end{aligned}
$$

Using (2.1) and (2.2) we obtain from above inequality

$$
\begin{aligned}
T(r, G) \leq & N_{k+1}\left(r, 0 ; f^{n}(a f+b)\right)+k \bar{N}(r, \infty ; f)+T(r, G) \\
& +N_{k+1}\left(r, 0 ; g^{n}(a g+b)\right)-(n+1) T(r, g)+\bar{N}(r, \infty ; g) \\
& +O\{\log r\}+S(r, g) .
\end{aligned}
$$

Hence

$$
(n+1) T(r, g) \leq(2 k+2) T(r, f)+(k+3) T(r, g)+S(r, g) .
$$

Thus we obtain

$$
(n-3 k-4)\{T(r, f)+T(r, g)\} \leq S(r, f)+S(r, g),
$$


a contradiction as $n \geq 3 k+9$.

Subcase 2. Let $B \neq 0$ and $A \neq B$. Then from (3.9) we get $F=\frac{(B+1) G-(B-A+1)}{B G+(A-B)}$ and so $\bar{N}\left(r, \frac{B-A+1}{B+1} ; G\right)=\bar{N}(r, 0 ; F)$. Proceeding as in Subcase 1 we obtain a contradiction.

Subcase 3. Let $B=0$ and $A \neq 0$. Then from (3.9) $F=\frac{G+A-1}{A}$ and $G=A F-(A-$ 1). If $A \neq 1$, we have $\bar{N}\left(r, \frac{A-1}{A} ; F\right)=\bar{N}(r, 0 ; G)$ and $\bar{N}(r, 1-A ; G)=\bar{N}(r, 0 ; F)$. So by Lemma 2.10 we have $n \leq 3 k+6$, a contradiction. Thus $A=1$ and hence $F=G$. Now using the same technique as used in Case 1 we can obtain (3.6) which by Lemma 2.11 gives $f=g$.

Case 3. Let $l=0$ and $H \not \equiv 0$. Using Lemma 2.5 and (3.2) we obtain from (3.3)

$$
\begin{aligned}
(n+1) T(r, f) \leq & N_{2}(r, 0 ; G)+N_{2}(r, \infty ; F)+N_{2}(r, \infty ; G)+2 \bar{N}(r, 0 ; F) \\
& +\bar{N}(r, 0 ; G)+N_{k+2}\left(r, 0 ; f^{n}(a f+b)\right)+2 \bar{N}(r, \infty ; F) \\
& +\bar{N}(r, \infty ; G)+O\{\log r\}+S(r, f)+S(r, g) \\
\leq & N_{k+2}\left(r, 0 ; f^{n}(a f+b)\right)+N_{k+2}\left(r, 0 ; g^{n}(a g+b)\right) \\
& +2 N_{k+1}\left(r, 0 ; f^{n}(a f+b)\right)+N_{k+1}\left(r, 0 ; g^{n}(a g+b)\right) \\
& +(2 k+4)(\bar{N}(r, \infty ; f)+(2 k+3) \bar{N}(r, \infty ; g)) \\
& +O\{\log r\}+S(r, f)+S(r, g) \\
\leq & {[5 k+11-(2 k+4) \Theta(\infty, f)-\varepsilon] T(r, f)+[4 k+8} \\
& -(2 k+3) \Theta(\infty, g)-\varepsilon] T(r, g)+O\{\log r\}+S(r, f)+S(r, g) \\
\leq & {[9 k+19-(2 k+3)(\Theta(\infty, f)+\Theta(\infty, g))} \\
& -\min \{\Theta(\infty, f), \Theta(\infty, g)\}+2 \varepsilon] T(r)+S(r) .
\end{aligned}
$$

Similarly

$$
\begin{aligned}
(n+1) T(r, g) \leq & {[9 k+19-(2 k+3)(\Theta(\infty, f)+\Theta(\infty, g))} \\
& -\min \{\Theta(\infty, f), \Theta(\infty, g)\}+2 \varepsilon] T(r)+S(r) .
\end{aligned}
$$

From (3.11) and (3.12) we get

$$
\begin{aligned}
& {[n-9 k-18+(2 k+3)(\Theta(\infty, f)+\Theta(\infty, g))} \\
& +\min \{\Theta(\infty, f), \Theta(\infty, g)\}-2 \varepsilon] T(r) \leq S(r),
\end{aligned}
$$

contradicts with the facts that $n \geq 9 k+18, \Theta(\infty, f)+\Theta(\infty, g)>4 / n$ and $\varepsilon>0$ be arbitrary. We now assume that $H=0$. Then proceeding in a similar manner as in Case 2 we obtain $f=g$. This completes the proof of the theorem.

Proof of Theorem 1.2. Noting that $\bar{N}(r, \infty ; f)=0, \bar{N}(r, \infty ; g)=0$ and using Lemma 2.9 instead of Lemma 2.8 and proceeding in the like manner as the proof of Theorem 1.1 we obtain the result of the theorem. 


\section{References}

[1] A. Banerjee, Uniqueness of certain nonlinear differential polynomials sharing 1-points, Kyungpook Math. J., 51(2011), 43-58.

[2] A. Banerjee, Meromorphic functions sharing one value, Int. J. Math. Math. Sci., 22(2005), 3587-3598.

[3] M. L. Fang, Uniqueness and value sharing of entire functions, Comput. Math. Appl., 44(2002), 823-831.

[4] C. Y. Fang and M. L. Fang, Uniqueness of meromorphic functions and differential polynomials, Comput. Math. Appl., 44(2002), 607-617.

[5] W. K. Hayman, Picard values of meromorphic Functions and their derivatives, Ann. of Math., 70(1959), 9-42.

[6] W. K. Hayman, Meromorphic Functions, The Clarendon Press, Oxford (1964).

[7] I. Lahiri, Weighted value sharing and uniqueness of meromorphic functions, Complex Var. Theory Appl., 46(2001), 241-253.

[8] I. Lahiri, Value distribution of certain differential polynomials, Int. J. Math. Math. Sc., 28(2001), 83-91.

[9] I. Lahiri, On a question of Hong Xun Yi, Arch. Math. (Brno), 38(2002), 119-128.

[10] I. Lahiri and S. Dewan, Value distribution of the product of a meromorphic function and its derivative, Kodai Math. J., 26(2003), 95-100.

[11] J. D. Li, Uniqueness of meromorphic functions and differential polynomials, Int. J. Math. Math. Sci., 2011, Article ID 514218, 12 pages, doi:10.1155/2011/514218.

[12] W. C. Lin and H. X. Yi, Uniqueness theorems for meromorphic function concerning fixed points, Complex Var. Theory Appl., 49(2004), 793-806.

[13] H. Y. Xu, C. F. Yi and T. B. Cao, Uniqueness of meromorphic functions and differential polynomials sharing one value with finite weight, Ann. Polon. Math., 95(2009), 51-66.

[14] C. C. Yang, On deficiencies of differential polynomials II, Math. Z., 125(1972), 107112 .

[15] L. Yang, Value Distribution Theory, Springer- Verlag, Berlin, 1993.

[16] H. X. Yi and C. C. Yang, Uniqueness Theory of Meromorphic Functions, Science Press, Beijing, 1995.

[17] J. L. Zhang, Uniqueness theorems for entire functions concerning fixed points, Comput. Math. Appl., 56(2008), 3079-3087.

[18] J. L. Zhang and L. Z. Yang, Some results related to a conjecture of $R$. Bruck, J. Inequal. Pure Appl. Math., 8(2007), Art. 18. 